\title{
Optimization of Tooling Design for Hot Mandrel Bending of Pipe Elbows
}

\author{
Uwe Diekmann ${ }^{1, \mathrm{a}}{ }^{*}$, Werner Homberg ${ }^{2, \mathrm{~b}}$, Jens Prehm ${ }^{1, \mathrm{c}}$, Tim Rostek $^{2, \mathrm{~d}}$, \\ Nils Schönhoff ${ }^{3, \mathrm{e}}$, Dmitri Tabakajew ${ }^{2, \mathrm{f}}$, Andreea Trasca ${ }^{1, \mathrm{~g}}$ and Haris Uysal ${ }^{3, \mathrm{~h}}$ \\ ${ }^{1}$ Metatech $\mathrm{GmbH}$, Lünener Straße 211/212, 59174 Kamen, Germany \\ ${ }^{2}$ Paderborn University, Department of Forming and Machining Technology (LUF), \\ Warburger Str. 100, 33098 Paderborn, Germany \\ ${ }^{3}$ Lindemann $\mathrm{GmbH} \&$ Co. KG, Orion-Eisenverarbeitung, Albert-Schweitzer-Str. 179, \\ 32257 Bünde, Germany \\ auwe.diekmann@metatech.pro, bwh@luf.uni-paderborn.de, ${ }^{\mathrm{c}}$ jens.prehm@metatech.pro, \\ dtr@luf.uni-paderborn.de, eschoenhoff@lindemann-buende.de, ${ }^{\mathrm{f}} \mathrm{dt} @ l u f . u n i-p a d e r b o r n . d e$, \\ gandreea.trasca@metatech.pro, hh.uysal@lindemann-buende.de
}

Keywords: Hot Forming, Pipe Bending, Numerical Simulation

Abstract. This paper presents the finite element model developed for the simulation of pipe elbow production by the so-called 'Hamburg process' in order to improve productivity and resource efficiency. To optimize the tooling design, a sensitivity analysis of the tool parameters that influence the quality of pipe elbows, such as mandrel height and length, is conducted. Using numerical simulations, it is possible to determine an optimized tool geometry for the production of specific pipe elbow dimensions. Furthermore, as a result of the experiments and numerical simulations conducted, it is possible to increase the production velocity of the serial plant. Along with deformation, damage models are included in simulations in order to identify the right process boundaries. Finally, an experimentally validated model is developed for increasing resource efficiency in pipe elbow fabrication.

\section{Introduction}

Pipe elbows are widely used in pipeline construction and process engineering. The Hamburg process is a manufacturing method for the production of pipe elbows through hot forming. Pipe elbows produced in this manner have a nearly uniform wall thickness. The production process consists of a bending mandrel and a feeding unit (Fig. 1) which pushes the pipe sections over the mandrel [1].

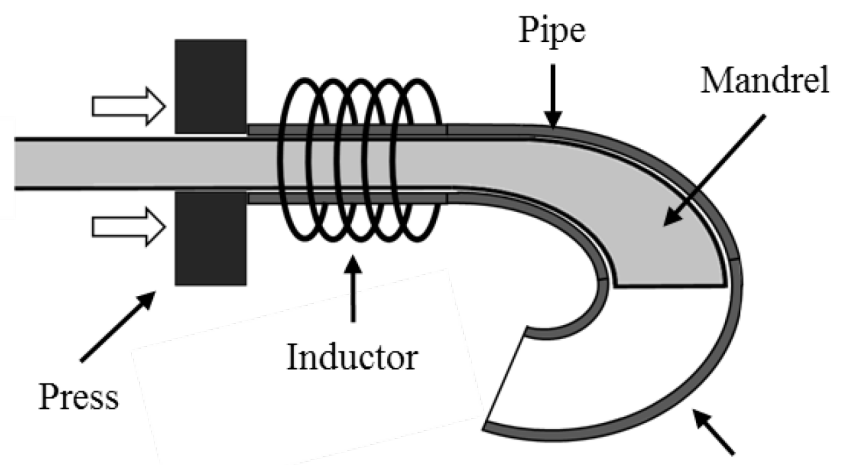

Elbow

Fig. 1. Production of pipe elbows with the Hamburg process

In order to increase the energy consumption and resource efficiency of this process, the process limits are investigated experimentally with a view to achieving a speed increase combined with temperature control. As a result of optimization calculations, the weight of the mandrel is reduced, on the one hand, and the amount excess material in the pipe is minimized, on the other. In the end, a considerable increase in productivity is achieved, accompanied by a higher efficiency in economic and ecological terms. 


\section{Hamburg Process}

In order to optimize the Hamburg process, experiments were carried out on a serial plant for seamless pipe elbows of different dimensions from steel P235, and the process and boundary conditions were analyzed. The results indicated that the production speed can be increased in proportion to the power of the inductor. If this is done, the temperature of the pipe elbows and the pressure on the pipes, or the load on the tools and plant respectively, remains nearly constant (Fig. 2a). In addition, the measured characteristics of pipe elbows, like wall thickness and outer diameter, hardly undergo any change [2].
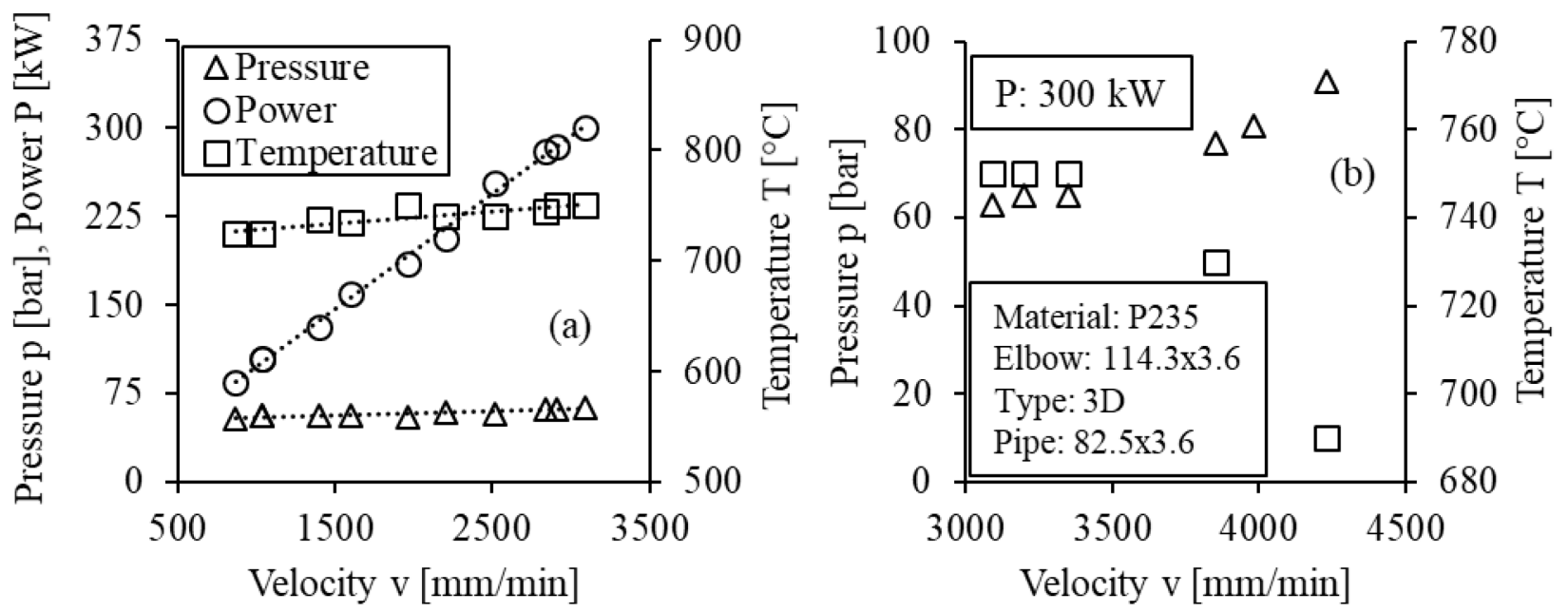

Fig. 2. Process parameters during the production of pipe elbows on a serial plant with a variable (a) and constant (b) power of the inductor

An increase in the production speed with an unchanged inductor power, by contrast, leads to a significant rise in the load on the tools and to a reduction in the measured temperature on the pipe elbows (Fig. 2b). Hence the right process window has to be selected here, since damage to the pipes can be expected [3] [4]. For this reason, the simulation of damage and failure was included in the analysis.

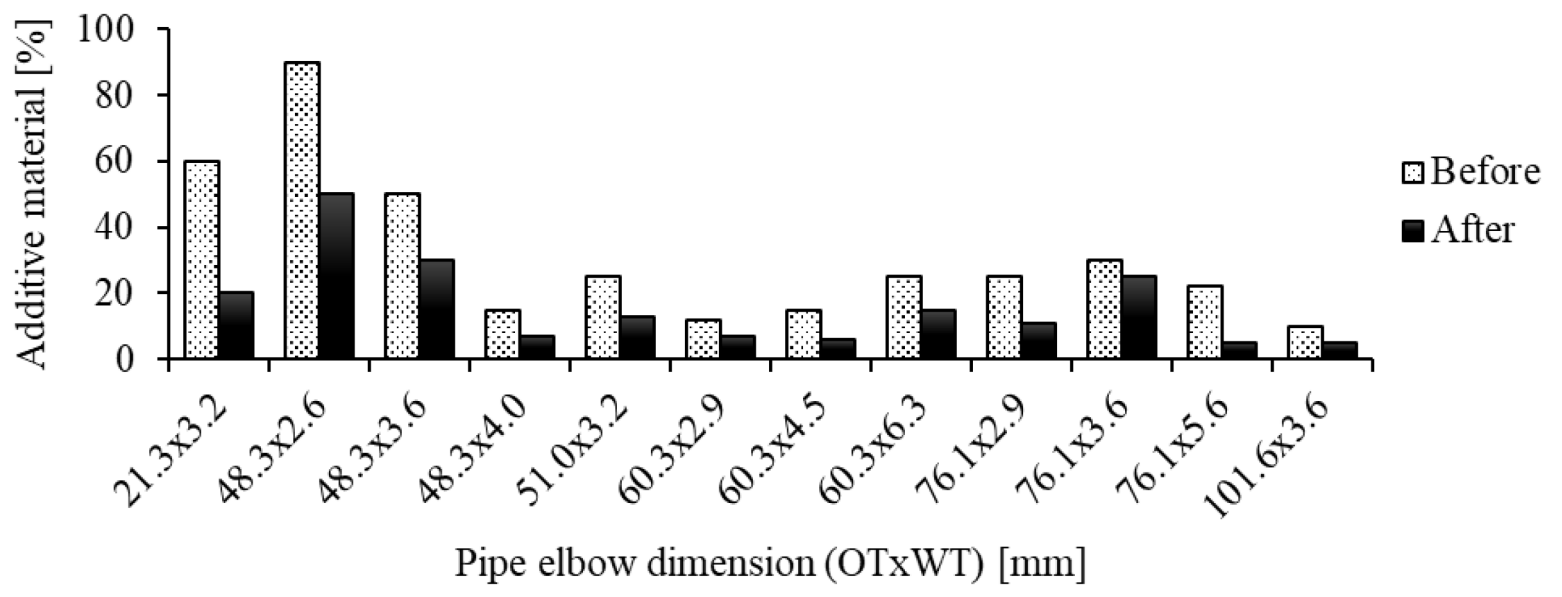

Fig. 3. Additive material (scrap) for different pipe elbow dimensions

In a further study, the excess material of the pipe sections needed to produce the elbows was analyzed. Here, it was established that the pipe sections are too long at the moment and can be shortened without any change in the output quantity of pipe elbows (Fig. 3). A significant reduction in raw material consumption and the production costs of pipe elbows are thus achieved. 


\section{Simulation Model of the Investigated Process}

The simulation of the Hamburg process and the improvement of the bending mandrel geometry were achieved using numerical models in Abaqus [5]. The numerical model includes a bending mandrel and a press plate that are defined as rigid bodies. The forming of three pipe sections is simulated, whereby only the second pipe section is analyzed. The first pipe section is needed to generate backpressure on the following pipe sections, and the third pipe section is required to push the front pipe section completely over the mandrel (Fig. 4).

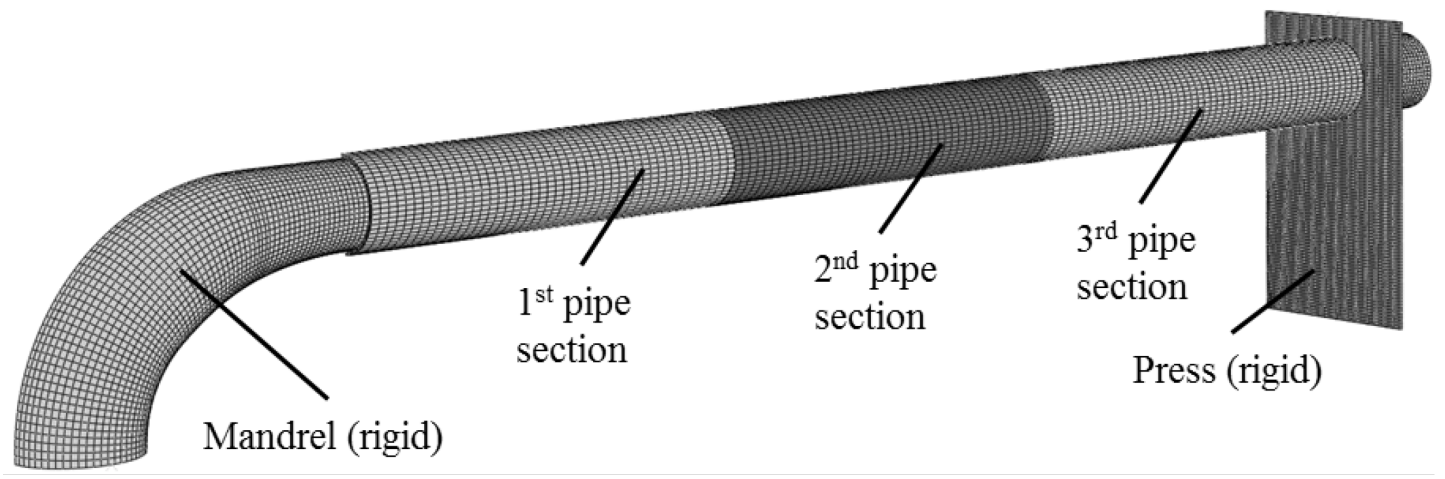

Fig. 4. Numerical model of Hamburg process

On the basis of the results from the process analysis performed, the production speed was drastically increased to $1200 \mathrm{~mm} / \mathrm{sec}$. Furthermore, it was assumed that the forming process is isothermal at $800{ }^{\circ} \mathrm{C}$. The validation of the numerical simulation was conducted by means of results from an optical forming analysis of pipe elbows using the GOM Argus software. The results have shown that the FE model depicts the production process with sufficient accuracy [2].

Throughout the modeling process, experimental flow stress data are used. Other material data needed for simulations (E-modulus, density, Poisson's Ratio, etc.) are computed using the JMatPro software. For purposes of employing measured data (which inherently show noise and oversampling) in more sophisticated models or as a CAE input, the raw data are manipulated in EDA software [7]. This means they are cleaned, resampled and averaged with special tools in EDA. The new, resampled data can now be used in modeling, for example, as suitable data for finding the Johnson parameters needed for damage simulations. In general, EDA permits more data from different measurements or simulations to be imported, manipulated and compared in this way.

\section{Optimization of the Mandrel Geometry}

The bending mandrel geometry currently used for the production of pipe elbows is based on the experience of design engineers. The difficulty with this is that there is a large number of possible parameters for the bending mandrel that can be changed. With the FE model that is introduced, different bending mandrel geometries can be tested in a highly cost-efficient manner and thus help to select the best bending mandrel geometry for individual elbow dimensions. To optimize the mandrel geometry, the three parameters of length, height and the angle of the area with no expansion are varied by comparison to the reference geometry, because previous experience has shown that these parameters have the biggest influence on the quality of pipe elbows (Fig. 5). Here, for example, the length of the bending mandrel was successively shortened, leading to a faster expansion of the pipe section. 


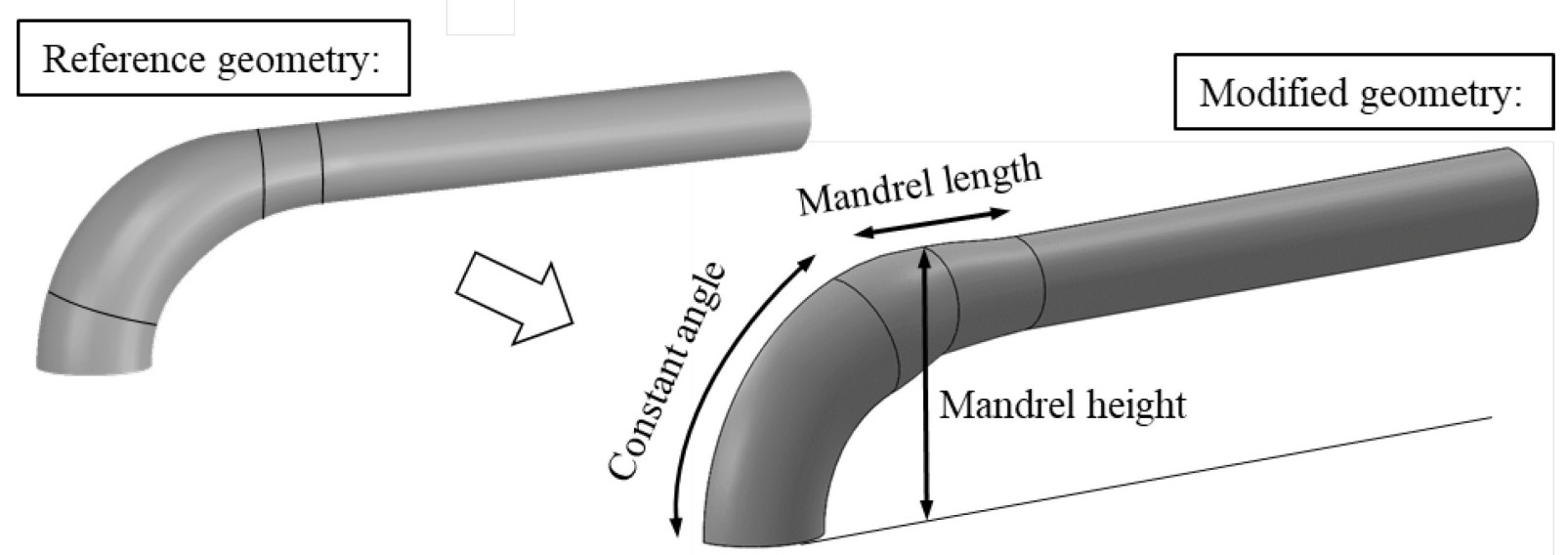

Fig. 5. Geometry parameters of the bending mandrel

An extension of the bending mandrel, by contrast, results in the expansion of the pipe section over a longer distance. The influence of these changes on the average outer diameter and the average wall thickness of the pipe elbows produced is shown in Fig. 6.

For the pipe elbow with target dimensions of $114.3 \mathrm{~mm} \times 3.6 \mathrm{~mm}$ and an original mandrel length of about $100 \mathrm{~mm}$, an extension of the expansion area leads to an increase in the average outer diameter of the pipe elbow and its standard deviation. For the average wall thickness, by contrast, a major reduction in the mandrel length leads to a deterioration of the values. This behavior can be explained by a higher backpressure acting on the pipe sections for the shortened bending mandrel and a very high deformation of the pipe sections in a relatively small area, which is typical for the Hamburg process.
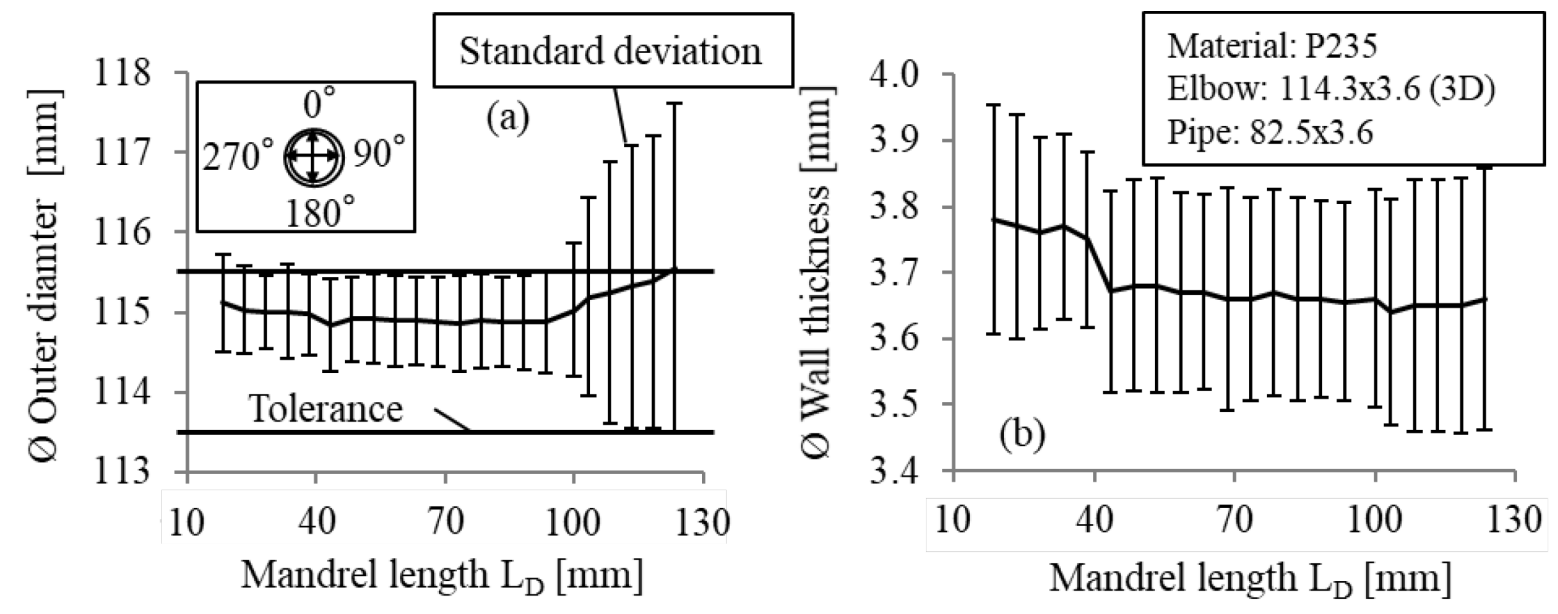

Fig. 6. Influence of changed mandrel length on (a) average outer diameter and (b) average wall thickness of pipe elbow

Another important factor for specifying the quality of the pipe elbows produced is a pipe elbow angle that is within the tolerance range without secondary processing. Ideally, in the production of a $90^{\circ}$ pipe elbow, this angle will be achieved from the shortest possible pipe section without any scrap. The value of the pipe elbow angle is based on the ovality of the pipe elbow that is calculated from the maximum and minimum outer diameter of the pipe elbow. Fig. 7 shows that a shortened bending mandrel considerably improves the attainable pipe elbow angle within the tolerance range as well as the ovality of the pipe elbow. 


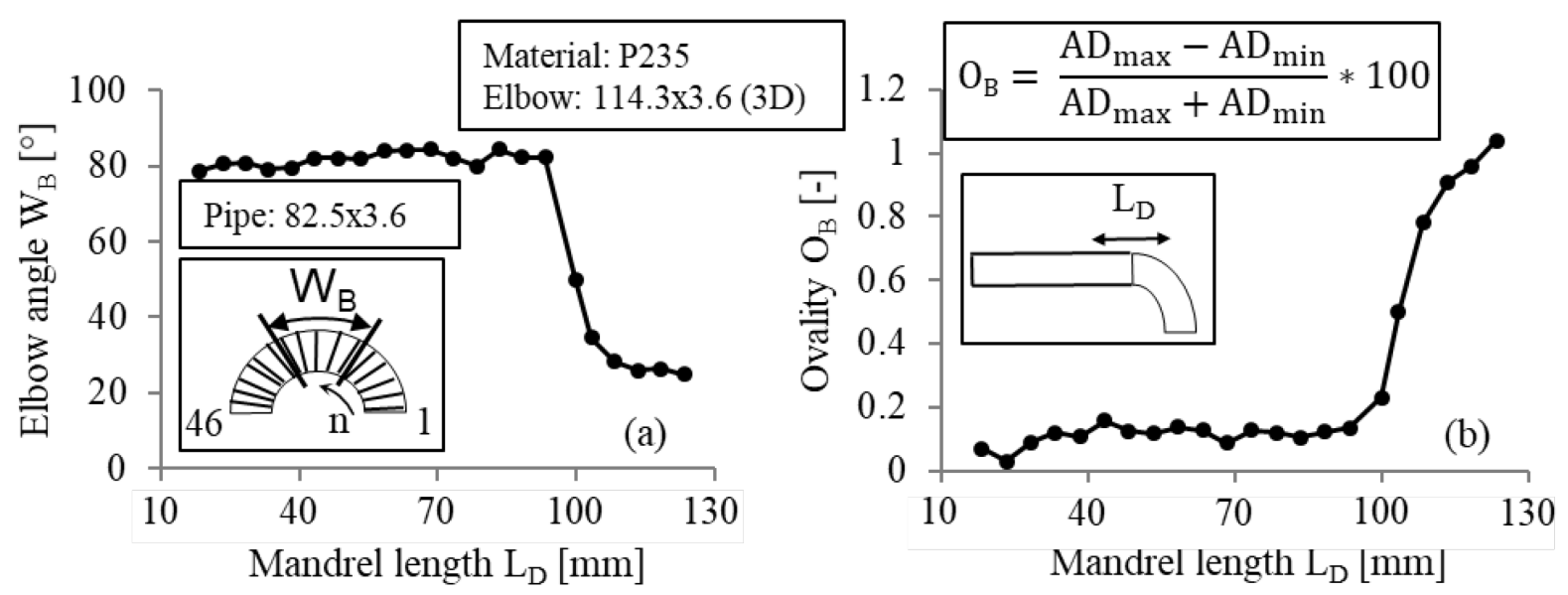

Fig. 7. Influence of changed mandrel length on (a) elbow length and (b) pipe elbow ovality

\section{Damage Modeling}

For the production of stainless steel pipe elbows, in particular, the temperature is of crucial importance to prevent cracking. For this reason, a model for the prediction of ductile damage has also been implemented. Here, the simplified Johnson-Cook (J-C) damage model integrated in LSDYNA was used [6]. In the simplified J-C model, the temperature and strain rate dependence of the stress function are not accounted for, and failure is supposed to occur when the strain exceeds the effective plastic failure strain, which is a function of the reduction in area. This shows the particular importance of failure modeling in stainless steels, as the effective plastic failure strain is only about half of the low alloyed steels. Fig. 8a shows the failure and creasing of stainless steel pipes in operation, while Fig. 8 b shows the corresponding simulation. In spite of the simplicity of the J-C model, the results of damage simulations show good agreement with those from measurements.
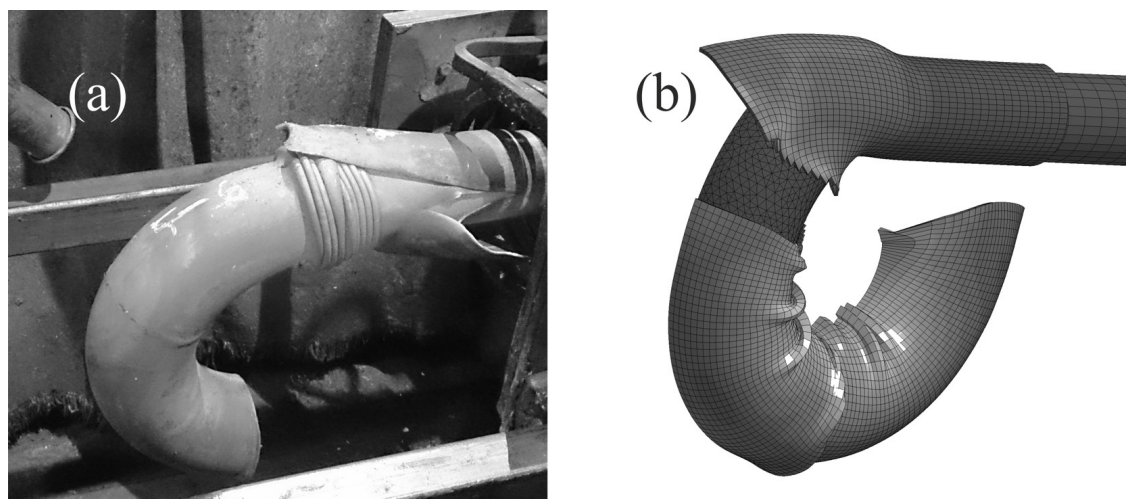

Fig. 8. Failure and creasing of stainless steel pipes in operation (a) and (b) corresponding simulation

\section{Optimization Tool}

An initial prototype of an optimization tool was created based on experience with the tasks described above:

- Simplified parametrical mandrel design based on the elbow geometry

- Selection from a set of 3 material models

- Automated pre-processing and calculation

The tool developed is used for determining the optimum bending mandrel geometry without technical knowledge of FEM modeling of hot-forming processes. The input parameters needed are the target dimension (e.g. outer diameter, type of construction, wall thickness) and target material. A parametric model, generated in the background, is subjected to a multi-stage optimization. Here, the results of extensive parameter studies covering the entire technically usable geometrical dimensions are taken into account. These studies have been investigated on the basis of practical experience and 
are stored in a knowledge database. For this reason, it is possible to greatly limit the process window taken into consideration for the variation calculations. The final output is an optimized mandrel geometry as per the identified CTQ-parameters (critical to quality).

\section{Conclusion}

Materials and energy efficiency, the final tolerances of the product and the production speed are the areas of optimization for the mandrel bending of elbow pipes. Based on experiments and isothermal runs, it was shown that the geometry of the mandrel has a big influence on the quality of the pipes. A parametrical design of the mandrel geometry was created, and three CTQ parameters were identified: length, height and the angle of the area with no expansion. The influence of individual geometry parameters was analyzed by means of a numerical simulation model. Simulation runs also resulted in a performance map for minimized material losses. Another finding was that experiments have confirmed the possibility of achieving a significant increase in productivity by increasing the processing speed. However, the creasing and failure of pipes during operation became a problem. Material models including damage have thus been implemented in the deforming pipe simulations and the requisite parameters were identified using data from experiments (J-C parameters) and from material simulations (JMatPro). Finally, the consolidated findings were used to develop an optimization tool for end-users in production engineering in order to find the best mandrel geometries and process data for custom pipe elbows.

\section{Acknowledgements}

This research and development project is being funded by the European Fund for Regional Development (EFRE) and by the state of North Rhine-Westphalia and managed by the Project Management Agency Jülich (PTJ). The authors are responsible for the contents of this publication.

\section{References}

[1] H. Naoi, H. Takagi, N. Tsugawa, A. Hozumi, S. Kawanishi, S-T. Jang, M. Wada, T. Yamakawa, T. Kurita, H. Sakai, T. Maruyama, Effects of mandrel shape on deformation behavior for hot mandrel bending of elbows, La Metallurgia Italiana - n. 11-12/2010, pp. 18-22.

[2] D. Tabakajew, W. Homberg, Increasing the efficiency of hot mandrel bending of pipe elbows, International Conference on the Technology of Plasticity, ICTP 2017, 17-22 September 2017, Cambridge, United Kingdom.

[3] X. Lu, Q. Zhou, L. Huang, J. Liu, Numerical simulation and parameter optimization of hot pushing elbow pipe bending process, Applied Mechanics and Materials, Vol. 432, 2013, pp. 92-97.

[4] A. Rodriguez, M. Mezzetti, P. Fodor, R. Colas, Forming of seamless pipe fittings, Journal of Materials Processing Technology, Vol. 120, 2002, pp. 432-437.

[5] E. Salas-Zamarripa, M.P. Guerrero-Mata, R. Colas, J. Martinez, Finite element model simulation of hot forging of seamless elbow fittings, Proceedings of the Institution of Mechanical Engineers Vol. 219, Part L: Journal of Materials Design and Applications, 2005, pp. 85-90.

[6] G.R. Johnson, W.H. Cook, A Constitutive Model and Data for Metals Subjected to Large Strains, High Strain Rates, and High Temperatures, Proceedings 7th International Symposium on Ballistics, The Hague, 19-21 April 1983, pp. 541-547.

[7] U. Diekmann, A. Miron, A. Trasca, Hybrid modeling of Material Properties for Improved CAE-Simulations, Materials Science Forum, Vol. 854, 2016, pp. 163-166. 